\title{
A new twist in targeting malaria parasites
}

Malaria is transmitted among humans by mosquitoes carrying sporozoite forms of protozoan Plasmodium species. The sporozoites reproduce abundantly in the liver of an infected person, creating tens of thousands of merozoites in a structure called a schizont, which later ruptures, releasing the parasites into the bloodstream. Some parasites develop into gametocytes that, when ingested by another mosquito, progress through the stages of ookinete and oocyst before maturing into sporozoites and completing the protozoan life cycle.

Most antimalarial drugs currently in use are effective only against the blood stages of infection and are further beset by the emergence of resistant Plasmodium strains. To better address the prevention, treatment and eventual eradication of malaria, public health experts recommend the development of new antimalarial drugs that can target multiple life stages, that are active against drug-resistant Plasmodium strains and that are less likely to select for resistant mutations. The ideal medicines would also be suited to single-dose oral administration to help ensure treatment compliance and would have long half-lives to help prevent reinfection.

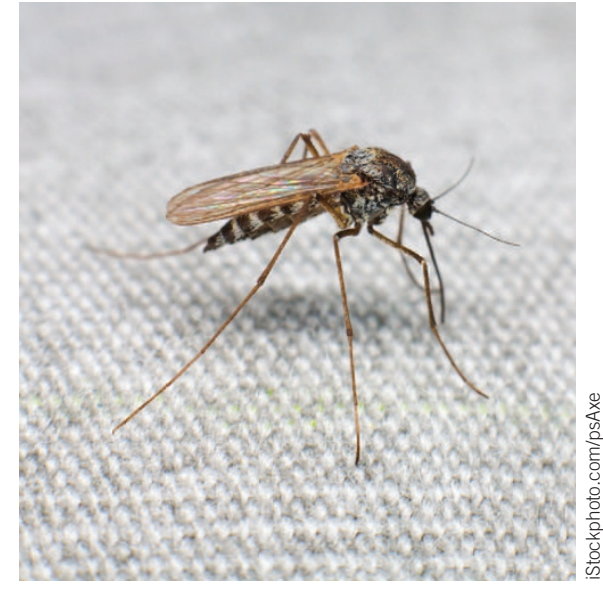

It's a tall order, to be sure. But an international group of biochemists recently reported a promising new candidate for malaria treatment called ELQ-300. It belongs to a class of compounds called quinolone3-diarylethers, modified from the quinolone drug endochin, which was first found to have antimalarial activity in the 1940s.

When administered to mice exposed to Plasmodium parasites, a single oral dose of ELQ-300 prevented infections from developing. In mice with existing malaria, four daily doses of ELQ-300 completely cured the infection (Sci. Transl. Med. 5, 177ra37; 2013). ELQ-300 targeted both the liver and blood stages of Plasmodium protozoa in hosts, as well as the life cycle stages involved in transmission via mosquito vectors (gametocytes, zygotes, ookinetes and oocysts). The drug was active against a Plasmodium falciparum strain that has a mutation associated with resistance to atovaquone, a currently used antimalarial medicine, and also boasted a much lower propensity to induce Plasmodium resistance. Combined with its high bioavailability, metabolic stability and oral efficacy, these characteristics make ELQ-300 an excellent candidate for further study. Michael K. Riscoe (Veterans Affairs Medical Center and Oregon Health \& Science University, Portland, OR) and Roman Manetsch (University of South Florida, Tampa), who led the development team, are investigating drug formulations and planning preclinical safety and toxicity studies. They expect to proceed to clinical trials of ELQ-300 within a few years.

Monica Harrington

\section{DOWN'S SYNDROME PROTEIN IDENTIFTED}

Researchers may have found the cause underlying the learning and memory impairments that characterize Down's syndrome. A protein called sorting nexin 27 (SNX27) in the brain is depleted in the brains of people with Down's syndrome, and restoring the protein in mice that model the disorder rescues the cognitive deficits (Nat. Med. published online 24 March 2013; doi:10.1038/nm.3117).

A team of researchers led by Huaxi Xu (Sanford-Burnham Medical Research Institute, La Jolla, CA) began by studying mice that are genetically deficient in SNX27 and noticed that they had several deficits characteristic of Down's syndrome patients, including fewer neurons in the hippocampus and cortex, defective synaptic functioning, and learning and memory impairments. The neurons of the mice also had reduced amounts of glutamate receptors, which are crucial for proper functioning of the neurons that underlie learning and memory. SNX27 helps keep glutamate receptors on the cell surface in neurons, and so without proper levels of SNX27, the neurons cannot function properly.

The researchers supposed that a SNX27 deficiency could be to blame in humans with Down's syndrome as well. They looked at levels of SNX27 in brains of humans with the condition and confirmed that there were significantly lower than normal levels of SNX27. There was also reduced expression of a protein called C/EBP $\beta$, which is necessary for SNX27 to be properly expressed. They discovered that C/EBP $\beta$ is negatively regulated by a microRNA called miR-155. This microRNA is encoded on chromosome 21; Down's syndrome patients have an extra copy of this chromosome, so they have increased levels of miR-155. In turn, the increased miR-155 leads to a decrease in C/EBP $\beta$ expression, which then reduces SNX27 expression, impairing localization of glutamate receptors in hippocampal and cortical neurons and resulting in cognitive deficits.

The team hopes to use this discovery to develop novel treatments for those living with Down's syndrome. They delivered human SNX27 into the brains of the Down's syndrome-modeling mice using a noninfectious virus delivery method. The increased levels of SNX27 in the hippocampus of the mice rescued their synaptic and cognitive deficits. Unfortunately, the same delivery system cannot safely be used in humans, so finding a treatment approach with SNX27 will not be quite so simple. The scientists are currently screening for small molecules that could be used as a drug to increase SNX27 levels. 